\title{
Nivel de conocimientos y prácticas sobre salud bucodental de estudiantes de posgrado de la Carrera de Enfermería de la Facultad de Ciencias de la Salud de la Universidad Católica de Asunción
}

\author{
* Nelson Osvaldo Santa Cruz González, Cesar Fernando Pérez Aquino, Nancy Villagra Rivera, Sofía \\ Achucarro Galeano
}

Universidad Católica de Asunción, Facultad de Ciencias de la Salud. Paraguay

Cómo referenciar este artículo/ How to reference this article:
Santa Cruz González NO, Pérez Aquino CF, Villagra Rivera N, Achucarro Galeano S. Nivel de conocimientos y prácticas sobre salud bucodental de estudiantes de posgrado de la Carrera de Enfermería de la Facultad de Ciencias de la Salud de la Universidad Católica de Asunción. Mem. Inst. Investig. Cienc. Salud. 2019; 17(2): 56-63

\begin{abstract}
RES U M E N
Los adultos jóvenes constituyen gran parte de la población paraguaya y se considera que los conocimientos y prácticas sobre salud bucodental están muy relacionados al nivel de instrucción, el objetivo de este estudio fue evaluar el nivel de conocimientos y prácticas sobre salud bucodental de estudiantes de posgrado de la Carrera de Enfermería de la Facultad de Ciencias de la Salud de la Universidad Católica de Asunción (CE FCSUCA), en el 2018. Estudio observacional descriptivo de corte transversal con componente analítico en el que se incluyeron a todos los estudiantes de posgrado entre mayo y junio de 2018. La recolección de datos se realizó mediante un cuestionario sobre salud bucodental autoadministrado y de carácter anónimo. Los datos se asentaron en una planilla electrónica, y fueron analizados utilizando el Programa estadístico IBMSSPS 23.0. La muestra estuvo constituida por 166 estudiantes, en su mayoría del sexo femenino $(75,3 \%)$, de entre 20 y 29 años $(54,8 \%)$, cursando algún programa de especialización $(57,8 \%$ y pertenecientes al área de la salud (94\%). El $57,83 \%$ y el $55,42 \%$ de los estudiantes presentaron conocimientos y prácticas de salud bucodental de nivel regular, respectivamente. Se encontró asociación entre nivel de conocimiento y de práctica y entre el nivel de conocimiento y la edad. Se concluye que los niveles de conocimientos y prácticas sobre salud bucodental en los estudiantes de posgrado fueron deficientes por lo que se propone la implementación de estrategias educativas de concienciación en el área.

Palabras clave: salud bucodental, educación, adultos.
\end{abstract}

\section{Level of knowledge and practices on oral health of graduate students of the Nursing Career of the Faculty of Health Sciences of the Catholic University of Asunción}

\begin{abstract}
A B S T R A C T
Young adults constitute a large part of the Paraguayan population and considering that oral health knowledge and practices are closely related to the level of education, the objective of this study was to evaluate the level of knowledge and practices on oral health of postgraduate students of the Nursing Career of the Faculty of Health Sciences of the Catholic University of Asunción (CE FCSUCA), in 2018. The design was descriptive, crosssectional with an analytical component in which all postgraduate students were included in May and June 2018. Data collection was carried out through a questionnaire on oral health self-administered and anonymous. The data were recorded in an electronic spreadsheet and analyzed using the IBMSSPS 23.0 Statistical Program. The sample consisted of 166
\end{abstract}

Fecha de recepción: junio 2019. Fecha de aceptación: julio 2019

*Autor correspondiente: Nelson Osvaldo Santa Cruz González. Universidad Católica de Asunción, Facultad de Ciencias de la Salud. Paraguay

Email: nelsanta88@gmail.com 
students, mostly female (75.3\%), between 20 and 29 years old $(54.8 \%)$, studying some specialization program (57.8\%) and belonging to the area of Health (94\%). $57.83 \%$ and $55.42 \%$ of the students presented knowledge and practices of oral health at the regular level, respectively. An association was found between the level of knowledge and practice and between the level of knowledge. It is concluded that the levels of knowledge and practices on oral health in the postgraduate students were insufficient, so the implementation of educational awareness strategies in the area is proposed.

Keywords: oral health, education, adults.

\section{INTRODUCCIÓN}

La salud bucodental es un componente indisociable de la salud general y forma parte del ciclo vital de las personas ${ }^{(1)}$, puede interferir en la calidad de vida, principalmente en la alimentación, la comunicación, el relacionamiento con los demás y la autoestima. Muchas enfermedades sistémicas se manifiestan primeramente en la boca por lo que es importante el diagnóstico precoz de los problemas de salud bucal ${ }^{(2-4)}$.

Las enfermedades bucodentales afectan con mayor frecuencia a los países en vías de desarrollo, y a la mayoría de la población adulta ${ }^{(5)}$. En Paraguay, el $63 \%$ de la población está representada por habitantes de entre 15 y 60 años de edad, considerados adolescentes y adultos jóvenes dentro de las etapas del ciclo vital ${ }^{(6)}$. Las enfermedades bucodentales más frecuentes en esta población son la caries y la enfermedad periodontal ${ }^{(7)}$.

La caries dental es una enfermedad infecciosa multifactorial, crónica, transmisible y prevenible, sin embargo y a pesar de los esfuerzos, es considerada una problemática en Salud Pública por su alta incidencia y prevalencia $^{(8)}$.

Las enfermedades periodontales son condiciones inflamatorias crónicas que afectan a los tejidos de soporte y protección del diente. Incluyen la gingivitis y la periodontitis crónica. Esta última puede causar la pérdida de dientes, pero hay que considerar que se inicia como una gingivitis y puede ser reversible aplicando medidas de prevención y tratamiento adecuadas ${ }^{(9-10)}$.

Entre los factores que intervienen para el surgimiento y la expansión de las enfermedades pueden considerarse los biológicos, el desarrollo económico, el desarrollo social y educativo de un país, así como la cultura y la tradición que regulan ciertos hábitos a nivel tanto individual como colectivo en una población ${ }^{(11)}$. Los conocimientos sobre salud bucodental inciden sobre el estado de salud de acuerdo a los factores anteriormente citados $^{(12)}$ y repercuten en las actitudes positivas o no hacia la prevención y cuidado de las enfermedades ${ }^{(13-14)}$.

El mayor conocimiento sobre salud bucodental crea condiciones favorables para mejorar las acciones de salud y modificar el comportamiento de las personas. Por lo tanto, los servicios de salud bucodental serían más concurridos ya desde las primeras manifestaciones de la enfermedad y se llevarían a cabo esquemas de promoción de la salud, prevención y atención de las afecciones que conllevaría a mejorar la esperanza y calidad de vida de las personas ${ }^{(15)}$.

El propósito de este estudio fue evaluar el nivel de conocimientos y las prácticas sobre salud bucodental de estudiantes de posgrado de la Carrera de Enfermería de la Facultad de Ciencias de la Salud de la Universidad Católica de Asunción en el año 2018.

\section{MATERIALES Y MÉTODOS}

Se llevó a cabo un estudio observacional descriptivo de corte transversal, con componente analítico, de tipo censal entre los meses de mayo y junio del 2018. La población de estudio estuvo constituida por 166 estudiantes de ambos sexos, de entre 20 y 60 años de edad, que dieron libre consentimiento para participar del mismo y que se encontraban matriculados en los cursos de posgrados de la Carrera de Enfermería de la Facultad de Ciencias de la Salud UCA en el periodo 2018. Se excluyeron los estudiantes de posgrado que estaban involucrados en el estudio, que se encontraban cursando más de un posgrado a la vez, con condición irregular y cuyos cursos no se iniciaban hasta abril.

Se utilizó un cuestionario autoadministrado y de carácter anónimo que incluía preguntas cerradas constituido por tres secciones: Datos sociodemográficos, prácticas de salud bucodental y conocimientos sobre salud bucodental. Las primeras dos secciones se adaptaron del estudio de Barrientos, Becerra, Parra \& Jouannet en adolescentes en el 
$2014^{(16)}$, y la tercera sección se adaptó del estudio de Dho(12) en adultos en el 2015 y Morel, Diaz y Quintana en el $2018^{(17)}$.

Los datos obtenidos se asentaron en planillas electrónicas para su tabulación y análisis estadístico utilizando el Programa IBM SSPS Statistics 23.0.

Se estableció un punto a las respuestas correctas y las no correctas o sin respuestas quedaron sin valor. De acuerdo a los puntajes obtenidos se determinaron los niveles de prácticas y conocimientos en: Nivel bueno $(\geq 80 \%)$, Nivel Regular $(\geq 50 \%)$ y Nivel Malo $(<50 \%)$ que finalmente fueron codificados con los números 3,2 y 1 respectivamente,

La asociación de variables se estableció mediante la prueba de chi cuadrado de Pearson a un nivel de significancia de 0,05.

\section{RESULTADOS}

Fueron incluidos 166 estudiantes de posgrado, en su mayoría del sexo femenino $(75,3 \%)$, de entre 20 y 29 años $(54,8 \%)$, pertenecientes al área de la salud $(94 \%)$, y que cursaban algún programa de especialización $(57,8 \%)$ (Ver Tabla 1 ).

Tabla 1: Datos sociodemográficos de estudiantes de posgrado CE FCS UCA 2018. n: 166

\begin{tabular}{lll}
\hline & Frecuencia & Porcentaje \\
\hline Edad & 91 & \\
$20-29$ años & 51 & 54,8 \\
$30-39$ años & 18 & 30,7 \\
$40-49$ años & 6 & 10,8 \\
$50-60$ años & & 3,6 \\
\hline Sexo & 41 & \\
Masculino & 125 & 24,7 \\
Femenino & & 75,3 \\
\hline Profesión & 156 & 94 \\
Área de la Salud & 10 & 6 \\
Otras áreas & & \\
\hline Programa de Posgrado & 32 & 19,3 \\
Diplomado & 24 & 14,5 \\
Capacitación & 57,8 \\
Especialización & 96 & 8,4 \\
Maestría & 14 &
\end{tabular}

El nivel de conductas o prácticas sobre su salud bucodental fue regular en el $55,42 \%$ o malo $(19,88 \%)$. El nivel de conocimiento sobre salud bucodental fue regular en el $57,83 \%$ o malo $(31,93 \%)$ de los participantes (Figura 1 ).

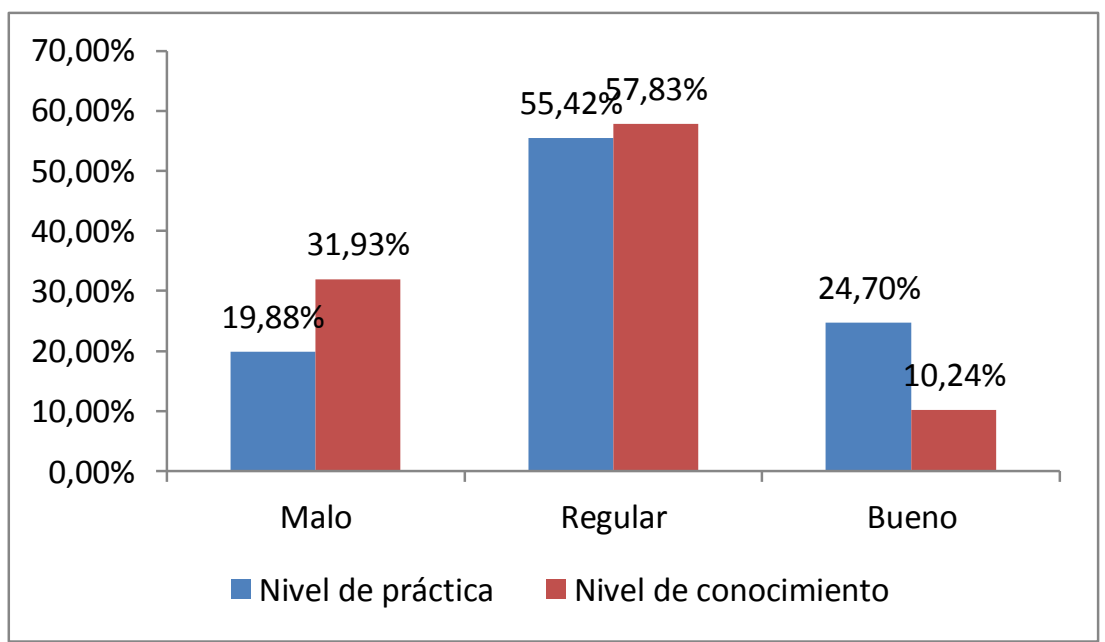

Figura 1: Nivel de conocimientos y prácticas sobre salud bucodental de estudiantes de posgrado CE FCS UCA 2018. $n: 166$ 
Los estudiantes refirieron no utilizar cepillo dental para su higiene bucal en $0,6 \%$, que la duración del cepillado fue menor a 2 minutos en $61,4 \%$, que $70,5 \%$ consumía azúcares entre las comidas y que $42,2 \%$ no se cepilla los dientes después del desayuno (Tabla 2 ).

Tabla 2: Prácticas sobre salud bucodental de estudiantes de posgrado CE FCS UCA 2018 n: 166

\begin{tabular}{lll}
\hline & Si & No \\
\hline Elementos de higiene bucal utilizados & & \\
Cepillo dental & $99,4 \%$ & $0,6 \%$ \\
Pasta dental & $93,4 \%$ & $6,6 \%$ \\
Hilo dental & $68,1 \%$ & $31,9 \%$ \\
Enjuagues & $63,9 \%$ & $36,1 \%$ \\
Otros aditamentos & $7,2 \%$ & $92,8 \%$ \\
\hline Cambio del cepillo dental & & \\
Cada 3 meses & $74,1 \%$ & $25,9 \%$ \\
Cada 6 meses o más & $25,9 \%$ & $74,1 \%$ \\
\hline Tiempo de duración del cepillado & $38,6 \%$ & $61,4 \%$ \\
Menos de 2 minutos & $61,4 \%$ & $38,6 \%$ \\
2 minutos o más & & \\
Consumo de azúcares en el día & $70,5 \%$ & $29,5 \%$ \\
Entre las comidas & $29,5 \%$ & $70,5 \%$ \\
Durante las comidas & & \\
\hline Momentos del cepillado dental & $3 \%$ & $97 \%$ \\
Al despertarse & $57,8 \%$ & $42,2 \%$ \\
Después del desayuno & $77,7 \%$ & $22,3 \%$ \\
Después del almuerzo & $94 \%$ & $6 \%$ \\
Antes de acostarse & \multicolumn{2}{|c}{} \\
\hline Control odontológico & $76,9 \%$ & $24,9 \%$ \\
Cada un año o menos & $24,1 \%$ & $76,9 \%$ \\
\hline Cada 2 años o por urgencia & & \\
\hline
\end{tabular}

Los estudiantes reconocieron a la caries como principal enfermedad bucodental de los adultos, no así a la gingivitis y la enfermedad periodontal en $59 \%$ y $89,2 \%$ respectivamente. Indicaron como causa de la caries principalmente a la mala higiene bucal ( $97 \%$ ) pero no reconocieron en $76,5 \%$ de los casos que el flúor es un factor protector ante la caries. No reconocieron, además, que el hábito de fumar puede causar gingivitis $(83,1 \%)$ y que las maneras de prevenirlas pueden ser consumiendo frutas con fibras $(77,7 \%)$ y utilizando hilo dental $(67,5 \%)$ (Ver Tabla 3$)$

Tabla 3: Conocimientos sobre salud bucodental de estudiantes de posgrado CE FCS UCA 2018. $n: 166$

\begin{tabular}{lll}
\hline & Si & No \\
\hline Las siguientes enfermedades afectan a gran parte de la población adulta & $95,8 \%$ & $4,2 \%$ \\
Caries & $41 \%$ & $59 \%$ \\
Gingivitis & $10,8 \%$ & $89,2 \%$ \\
Periodontitis & & \\
\hline Factores que favorecen la formación de caries & $97 \%$ & $3 \%$ \\
Mala higiene bucal & $20,5 \%$ & $79,5 \%$ \\
Bacterias & $28,9 \%$ & $71,1 \%$ \\
\hline Consumo de hidratos de carbono & & \\
\hline Factores protectores ante la caries & $63,9 \%$ & $36,1 \%$ \\
Consulta odontológica & $23,5 \%$ & $76,5 \%$ \\
Utilización de flúor & $89,8 \%$ & $10,2 \%$ \\
Cepillado dental después de cada comida & $41 \%$ & $59 \%$ \\
\hline Uso diario de hilo dental & $97 \%$ & $3 \%$ \\
\hline La caries puede producir la perdida de diente & $88 \%$ & $12 \%$ \\
\hline Es normal el sangrado de las encías al cepillado dental & $79,5 \%$ & $20,5 \%$ \\
\hline La gingivitis es una reacción de defensa del cuerpo ante la placa bacteriana & & \\
\hline Factores que contribuyen a la gingivitis & $72,3 \%$ & $27,7 \%$ \\
Mala higiene bucal & $54,2 \%$ & $45,8 \%$ \\
Bacterias & $16,9 \%$ & $83,1 \%$ \\
\hline Hábito de fumar & &
\end{tabular}




\begin{tabular}{|c|c|c|}
\hline actores que ayudan a prevenir la gingivitis & & \\
\hline Consulta periódica al odontólogo & $63,9 \%$ & $36,1 \%$ \\
\hline Cepillado dental diario & $81,3 \%$ & $18,7 \%$ \\
\hline Consumo de frutas con fibras & $22,3 \%$ & $77,7 \%$ \\
\hline Uso diario del hilo dental & $32,5 \%$ & $67,5 \%$ \\
\hline $\begin{array}{l}\text { Las enfermedades periodontales pueden ser coadyuvantes en } \\
\text { enfermedades sistémicas }\end{array}$ & $92,2 \%$ & $7,8 \%$ \\
\hline Los dientes permanentes pueden durar toda la vida & $66,9 \%$ & $33,1 \%$ \\
\hline
\end{tabular}

Se encontró asociación entre el nivel de conocimiento y la edad $(0,013)$. Las demás variables sociodemográficas no presentaron asociaciones con los niveles de conocimientos y prácticas.

Tabla 4: Nivel de conocimientos sobre salud bucodental según edad CE FCS UCA. 2018. $\mathrm{n}: 166$

\begin{tabular}{lllll}
\hline Edad & Total & \multicolumn{3}{l}{ Nivel de conocimientos } \\
\cline { 2 - 5 } & \multicolumn{5}{c}{ Malo } & Regular & Bueno \\
\hline $\mathbf{2 0 - 2 9}$ & 91 & $35(38,5 \%)$ & $48(52,7 \%)$ & $8(8,8 \%)$ \\
$\mathbf{3 0 - 3 9}$ & 51 & $15(29,4 \%)$ & $34(66,7 \%)$ & $2(3,9 \%)$ \\
$\mathbf{2 4 0}$ & 24 & $3(12,5 \%)$ & $14(58,3 \%)$ & $7(29,2 \%)$ \\
\hline Valor $\mathrm{p}=0,013$ & \multicolumn{5}{c}{}
\end{tabular}

Además, se encontró asociación entre el nivel de prácticas con respecto al nivel de conocimientos sobre salud bucodental $(0,001)$ (Tabla 4$)$.

Tabla 5: Nivel de prácticas en relación al nivel de conocimientos de los estudiantes de posgrado CE FCS UCA 2018. n: 166

\begin{tabular}{lllll}
\hline $\begin{array}{l}\text { Nivel de } \\
\text { conocimientos }\end{array}$ & Total & $\begin{array}{l}\text { Nivel de prácticas } \\
\text { Malo }\end{array}$ & Regular & Bueno \\
\hline Malo & 52 & $19(11,45 \%)$ & $23(13,83 \%)$ & $10(6,02 \%)$ \\
Regular & 97 & $12(7,23 \%)$ & $65(39,16 \%)$ & $20(12,05 \%)$ \\
Bueno & 17 & $0(0 \%)$ & $11(6,63 \%)$ & $6(3,61 \%)$ \\
\hline
\end{tabular}

Valor $p=0,001$

\section{DISCUSIÓN}

Dentro de los hallazgos en esta investigación resalta que la población universitaria casi en su totalidad $(95,8 \%)$ reconoció la caries como enfermedad bucal de los adultos. En contraste con el estudio de Lawder et al. en $2008^{(18)}$ que encontraron que el $60 \%$ de la población, de nivel de escolaridad bajo, reconoció la caries como enfermedad bucal. Además de la caries, los participantes de este estudio reconocieron en el $40 \%$ de los casos a la gingivitis como enfermedad bucal de los adultos y apenas $10,8 \%$ a la periodontitis.

En Baltimore ${ }^{(14)}$ en un estudio realizado en el 2011 en adultos de bajos ingresos con apenas 12 años de escolaridad, el 91\% reconoció al consumo de azúcares como causa principal de la caries. Sin embargo, en el presente estudio el conocimiento de los hidratos de carbono como causa de caries fue solo del $28,9 \%$, siendo la más reconocida la mala higiene bucal. Esta discordancia puede deberse a que la palabra azúcar no estaba explícita en el cuestionario utilizado debido a las modificaciones realizadas para esta población adulta. Por otro lado, el $82 \%$ de los participantes en el estudio de Baltimore indicó que la caries podría prevenirse con el cepillado dental y uso del hilo dental, casi concordante con este trabajo donde el $89,9 \%$ reconoció como factor protector al cepillado dental después de cada comida, seguido por las consultas periódicas al odontólogo y el uso del hilo dental. Pero llama la atención que solo el 23,5\% identificó que el flúor es un factor protector, cuando que Mattos-Vela et al. ${ }^{(19)}$, en un estudio a padres y profesores de preescolares lo identificaron en un $74,8 \%$ y $73 \%$ respectivamente. Tales diferencias pueden deberse a que los estudiantes marcaron los factores protectores asociándolos a las prácticas que realizan regularmente. 
Con respecto al sangrado de las encías durante el cepillado, el $88 \%$ creía que es normal, pero consideraron en un porcentaje similar que la gingivitis es una reacción de defensa ante las bacterias de la placa bacteriana. Los factores más reconocidos que pueden ayudar a prevenirla son el cepillado dental diario y la consulta periódica al odontólogo y en un porcentaje mucho menor el uso del hilo dental y el consumo de frutas con fibras en apenas $32,5 \%$ y $22,3 \%$, respectivamente.

En general, en esta investigación se encontró que los estudiantes de posgrado de la Carrera de Enfermería de la Facultad de Ciencias de la Salud de la Universidad Católica de Asunción presentaban conocimientos regulares en $57,83 \%$ con respecto a la salud bucodental y prácticas también regulares en $55,42 \%$. Por lo tanto, los niveles superiores de educación en las personas no se asocian a los buenos conocimientos y buenas prácticas sobre salud bucodental.

En algunos estudios presentados con anterioridad se utilizaron escalas diferentes para medir los conocimientos y prácticas sobre salud bucodental, lo que dificulta la comparación con los resultados de la presente investigación. Benítez et al. ${ }^{(20)}$ encontraron que el $33 \%$ de los participantes presentaron un nivel de conocimiento suficiente y $8 \%$ de pácticas adecuadas. Nakagawa et al. ${ }^{(21)}$ en el 2015 hallaron que el $56 \%$ de los participantes presentaron niveles de prácticas desfavorables, y un nivel de conocimiento bajo en $58,75 \%$ de los casos.

Delgado \& Ayala ${ }^{(22)}$ en el 2012 midieron el nivel de conocimiento acerca de la salud bucal de los docentes de educación inicial y primaria en Perú, en el que encontraron un nivel regular en $53 \%$, datos similares a los que Núñez et al. ${ }^{(23)}$ hallaron en embarazadas en una clínica privada en Chile, con un nivel de conocimientos regular en un $64 \%$. En contraste, Rivadeneira, et al. ${ }^{(24)}$ encontraron un nivel malo de conocimientos sobre salud bucodental en pacientes de más de 15 años que pudieron corroborar por la presencia de enfermedades bucales en los participantes. Tal situación clínica también fue encontrada por Dho et al. ${ }^{(25)}$ al examen bucal en su estudio de 2013, no coincidente con el nivel de conocimientos que predominó en su estudio que resultó ser bueno.

En el 2015, Dho(12) encontró un nivel de conocimientos de salud bucodental aceptable en el que aquellos con nivel socioeconómico medio-bajo presentaron conocimientos significativamente menores que los niveles alto, medio-alto y bajo.

Como instrumento de recolección de datos, la muestra censal, puede considerarse un método eficaz y válido en la selección o reclutamiento de los participantes para lograr una muestra representativa de la población de estudio. Aunque, debido a la irregularidad de algunos estudiantes en los programas aplicados, al ausentismo en los días de aplicación del instrumento y a la alta tasa de deserción de los mismos en los primeros meses de estudio no se pudo acceder a la totalidad de matriculados.

A pesar de las posibles limitaciones mencionadas, el presente estudio proporciona una visión general de los conocimientos y prácticas de salud bucodental que presenta la población adulta con un nivel de instrucción elevado.

Considerando que esta investigación no contempló el examen clínico de los encuestados ni las actitudes de las personas hacia su salud bucodental, se recomienda agregar inspecciones bucales y la medición de las actitudes en futuros estudios, con el objeto de contrastar lo que las personas conocen y hacen para cuidar su salud bucal con respecto a su estado bucal general; y evidenciar las actitudes de las personas hacia su cuidado partiendo de la premisa que además de tener conocimientos, es necesario mantener y fomentar la actitud preventiva y positiva sobre la salud bucodental en todas las etapas del ciclo vital, con la cual se logren prácticas favorables y el goce de una buena salud bucodental y mejora de la calidad de vida.

Fuentes de financiación: los autores.

Declaración de conflicto de intereses: No existentes.

\section{REFERENCIAS BIBLIOGRÁFICAS}

1. Delgado R, Ayala G. Nivel de conocimiento sobre salud bucal de los docentes de educación inicial y primaria en los distritos rurales de huachos y capillas, provincia de Castrovirreyna-Huancavelica: (monografía en Internet)*. Lima, Universidad Nacional Mayor San Marcos; 2012 (acceso 10 de marzo de 2018). Disponible en http://cybertesis.unmsm.edu.pe/bitstrea 
$\mathrm{m} /$ handle/cybertesis/1127/Delgado_rl.pdf ?sequence $=1$

2. Rodríguez M, Arpajón $Y$, Herrera I. Autopercepción de salud bucal en adultos mayores portadores de prótesis estomatológica. Rev haban cienc méd (revista en Internet)*2016 (acceso febrero 2018); 15 (1). Disponible en http://scielo.sld.cu/scielo.php?script $=$ sci arttext\&pid=S1729-519X2016000100007

3. Saliba-Garbin C, Isper-Garbin A, MoreiraArcieri R, Saliba N, \& Gonçalves P. La Salud Bucal en la Percepción del adolescente. Rev de Salud Pública (revista en Internet)* 2009 (acceso febrero 2018); 11 (2) 268-77. Disponible en http://www.scielo.org.co/pdf/rsap/v11n2/ v11n2a11.pdf

4. Caballero CR, Flores JF, Arenas L. Salud bucodental relacionada a la calidad de vida: revisión crítica de los instrumentos de medición. Mem. Inst. Investig. Cienc. Salud (revista en Internet)* 2017 (acceso agosto 2018); 15 (2): 108-17. Disponible en:

http://scielo.iics.una.py/pdf/iics/v15n2/18 12-9528-iics-15-02-00108.pdf

5. Dirección General de Estadísticas Encuestas y Censos (sede Web) 2016 (acceso marzo de 2018). Encuesta permanente de hogares. Disponible en http://www.dgeec.gov.py/Publicaciones/Bi blioteca/PREPH2016/PRINCIPALES\%20RE SULTADOS\%20EPH\%202016.pdf

6. OMS (sede Web). Centro de prensa. Abril 2012 (acceso 20 de marzo de 2018). Salud Bucodental. Disponible en http://www.who.int/mediacentre/factshee ts/fs318/es/

7. Ministerio de Salud Pública y Bienestar Social (sede Web). Dirección de Salud Bucodental. noviembre de 2012 (acceso marzo de 2018). Guía de Abordaje Integral a la salud bucodental para el equipo de salud. disponible en http://www.mspbs.gov.py/bucodental/wpcontent/uploads/2013/04/Guia-

Abordaje_enero20131.pdf

8. Ministerio de Salud Pública y Bienestar Social. (sede Web). Julio 2017 [acceso marzo de 2018]. Encuesta Nacional de Salud Bucodental. Disponible en http://www.mspbs.gov.py/bucodental/enc uesta-nacional-salud-bucodental-2017

9. Flemmig T. Periodontitis. Ann Periodontol. (revista en Internet)* 1999 (acceso marzo de 2018); 32-38. Doi: 10.1902/annals.1999.4.1.32

10. Carvajal P. Enfermedades periodontales como un problema de salud pública: el desafío del nivel primario de atención en salud. Revista Clínica de Periodoncia, Implantologpia y Rehabiilitación Oral (revista en Internet)* 2016 (acceso marzo de 2018);9 (2), 85-216. Disponible en: https://www.elsevier.es/es-revista- revista-clinica-periodoncia-implantologiarehabilitacion-200-articulo-enfermedadesperiodontales-como-un-problemaS0718539116300337

11. Unfer B, Saliba O. Avaliação do conhecimento popular e práticas cotidianas em saúde bucal. Rev Saúde Pública (revista en Internet)*. Abril 2000 [acceso marzo 2018]; 34(2):190-5. Disponible

en http://www.scielo.br/scielo.php?script $=$ sci_arttext\&pid $=$ S0034-

$89102000000200014 \&$ Ing $=$ en. Doi: $10.1590 / S 0034-89102000000200014$

12. Dho MS. Conocimientos de salud bucodental en relación con el nivel socioeconómico en adultos de la ciudad de Corrientes, Argentina. Rev. Fac. Nac. Salud Pública (revista en Internet)* 2015 (acceso marzo 2018); 33(3): 361-369. Disponible en: http://www.scielo.org.co/pdf/rfnsp/v33n3 /v33n3a05.pdf

13. Gholami M, Pakdaman A, Jafari A, Virtanen JI. Knowledge of and attitudes towards periodontal health among adults in Tehran. East Mediterr Health J. (revista en Internet)*. 2014 [acceso marzo 2018]; 20 (3): 196-202. Disponible en http://applications.emro.who.int/emhj/v2 0/03/EMHJ_2014_20_3_196_202.pdf

14. Macek M, Manski M, Schneiderman M, Meakin S, Haynes D, Wells W, et al. Knowledge of Oral Health Issues Among Low-Income Baltimore Adults: A Pilot Study. J Dent Hyg (revista en Internet)*. 2011 (acceso marzo 2018); 85 (1): 49$56 . \quad$ Disponible en: https://www.ncbi.nlm.nih.gov/pmc/article S/PMC3612929/

15. Núñez J, Moya $P$, Monsalves MJ, Landaeta S. Nivel de Conocimiento de Salud Oral y Utilización de GES Odontológico en Puérperas Atendidas en una Clínica Privada, Santiago, Chile. Int. J. Odontostomat. (revista en Internet) *. 2013 (acceso Abril 2018); 7(1): 39-46. Disponible en: https://scielo.conicyt.cl/scielo.php?script= sci_arttext\&pid=S0718-

$38 \overline{1} \times 2013000100007 \&$ Ing=es. http://dx. doi.org/10.4067/S07

16. Barrientos C, Becerra P, Parra A, \& Jouannet J. Conocimiento, actitudes y prácticas en salud oral en adolescentes de 12 y 15 de la localidad de Fresia, año 2013. Rev Chil Salud Pública. (revista en Internet)*. 2014 (acceso marzo 2018); 18(2): 140-8. Disponible en: 10.5354/0719-5281.2014.31975

17. Morel M, Diaz C, Quintana E. Nivel de conocimientos sobre salud oral e índice de caries en adolescentes de una escuela de Pirayú, Paraguay. Pediatr. (Asunción). (revista en Internet)* 2018 (acceso 
noviembre 2018); 45(3):212-16.

Disponible en:

https://revistaspp.org/index.php/pediatria /article/view/467/414?fbclid=IwAR3_fIEm SifrUdt5dqrVCl1CQP7ikwbdKGKmgXTOa3_I JxC1UKbz3dSSzK0

18. Lawder J, Mendes Y, Da silva I, Andradei D, Rocha I, Rogalla T, et al. Conhecimento e Práticas em Saúde Bucal Entre Usuários de Serviços. Pesq Bras Odontoped Clin Integr. (revista en Internet)* 2008 (acceso febrero 2018); 8(3):321-326. Disponible en:

https://www.proteseedentistica.com.br/w p-content/uploads/2015/05/artigo_30.pdf.

19. Mattos-Vela MA, Carrasco-Loyola MB, Valdivia-Pacheco SG. Nivel de Conocimiento sobre Pasta Dental Fluorada en Padres y Profesores de Preescolares. Int. J. Odontostomat. (revista en Internet)* 2013 (acceso marzo 2019); 7(1):17-24. Disponible en:

https://scielo.conicyt.cl/scielo.php?script= sci_arttext\&pid=S0718-

$381 \times 2013000100003 \&$ Ing $=$ es. http://dx. doi.org/10.4067/S0718381X2013000100003.

20. Benítez G, Giménez ES. \& Pérez G. Conocimiento, Actitud y Prácticas sobre salud bucal de alumnos del Instituto Superior de Educación "Dr. Raúl Peña" en el año 2010. Facultad de Odontología UNA. Asunción; 2010.

21. Nakagawa $M$, Ojeda D, Caballero E \& Amarilla A. Conocimiento, actitud y práctica sobre salud bucal en alumnos del último año de Institutos de Formación Docente del Paraguay en el año lectivo 2015. Facultad de Odontología UNA. Asunción; 2015.
22. Delgado $R$, \& Ayala $G$. Nivel de conocimiento sobre salud bucal de los docentes de educación inicial y primaria en los distritos rurales de huachos y capillas, provincia de Castrovirreyna Huancavelica. Universidad Nacional Mayor de San MArcos. Facultad de Odontología (revista en Internet)* 2012 (acceso marzo 2018). Disponible

en http://cybertesis.unmsm.edu.pe/bitstrea $\mathrm{m} /$ handle/cybertesis/1127/Delgado_rl.pdf ?sequence $=1$

23. Núñez J, Moya $P$, Monsalves $M$, \& Landaeta S. Oral Health Level of Knowledge and Use of Dental GES in Puerperal Patients at a Private Clinic, Santiago, Chile. Int J Odontostomat. (revista en Internet)* 2013 (acceso marzo 2018); 7(1): 39-46. Disponible en https://scielo.conicyt.cl/scielo.php?script= sci_arttext\&pid=S0718-

$381 \times 2013000100007$

24. Rivadeneira A, Nazco L, Hernández Pérez M. \& Hernández Marín C. Nivel de conocimiento sobre salud buco-dental. Consultorio 1ro de marzo. Febrero-octubre 2009. Gaceta Médica Espirituana. (revista en Internet)* 2013 (acceso marzo 2018); 15(1). Disponible en: http://bvs.sld.cu/revistas/gme/pub/vol.15 .(1)_09/p9.html

25. Dho M, Vila V, \& Palladino A. (2013). Situación de salud bucal de pacientes mayores de 18 años. Cátedra Práctica Clínica Preventiva I, Facultad de Odontología. UNNE, Argentina, 2010. Revista Facultad de Odontología Universidad de Antioquia (revista en Internet)* 2013 (acceso marzo 2018); 24(2): 214-31. Disponible en http://aprendeenlinea.udea.edu.co/revistas/i ndex.php/odont/article/view/9502/13551 